Universidade Tecnológica Federal do Paraná - UTFPR

Campus Ponta Grossa - Paraná - Brasil

ISSN: 1981-3686 / v. 02, n. 01: p. 49-66, 2008

\section{Revista Brasileira de Tecnologia Agroindustrial}

\title{
PERCEPÇÃO TEMPORAL DE DOÇURA, AMARGOR E SABOR DE FRUTA EM NÉCTAR DE GOIABA ADOÇADO COM DIFERENTES ADOÇANTES
}

\section{TEMPORAL PERCEPTION OF SWEETNESS, BITTERNESS AND FRUITINESS ON GUAVA NÉCTAR SWEETNED WITH DIFERENT SWEETENERS}

\author{
*Carlos Alexandre Koguishi de Brito; Helena Maria André Bolini \\ *Laboratório de Análise Sensorial - Faculdade de Engenharia de Alimentos, Universidade Estadual de \\ Campinas - UNICAMP. Brasil. brito@,fea.unicamp.br
}

\section{Resumo}

Este trabalho objetivou determinar o comportamento das características temporais dos estímulos doce, amargo e sabor de fruta em néctar de goiaba (Psidium Guajava L.) adoçado com sacarose e cinco edulcorantes: sucralose, ciclamato/sacarina 2:1, acessulfame-k, aspartame e estévia (Stévia rebaudiana Bertoni), com doçura equivalente à solução de sacarose a 10\%. Foi utilizado o método tempo-intensidade (T-I) e as curvas tempo-intensidade para cada substância foram coletadas utilizando-se o programa "Sistema de Coleta de Dados Tempo-Intensidade - SCDTI" para Windows, onde os provadores registravam com auxílio do "mouse" a percepção de cada estímulo solicitado em função do tempo, para cada amostra. A amostra adoçada com estévia apresentou a maior intensidade máxima para os estímulos doce e amargo e essa característica persistiu por longo período, indicando a presença de doçura e de amargor residual intenso. A amostra com sacarose caracterizou-se pelo gosto doce limpo, sem residual amargo. Em relação ao estímulo sabor de fruta, a sacarose exibiu a maior intensidade máxima. Já o aspartame apresentou o maior tempo total de duração do estímulo, indicando potencialização do sabor de fruta por esse edulcorante em néctar de goiaba. O aspartame foi o edulcorante, cujo comportamento sensorial mais se aproximou ao da sacarose na análise tempointensidade.

Palavras-chave: goiaba, néctar, tempo-intensidade, edulcorante.

\section{Introdução}

$\mathrm{O}$ aspecto mais importante para a maioria dos consumidores são as características sensoriais, os estímulos químicos e físicos sentidos pelos consumidores através do aroma, da textura e do sabor dos alimentos (FORDE \& DELAHUNTY, 2004). Os testes sensoriais são importantes no controle de qualidade por serem capazes de identificar a presença ou ausência de diferenças perceptíveis, definirem 
as características sensoriais importantes de um produto de forma rápida e serem capazes de detectar particularidades dificilmente detectadas por outros métodos analíticos (MUÑOZ, CIVILLE \& CARR, 1992). Na avaliação sensorial clássica, como análise descritiva quantitativa ou outras formas de avaliação utilizando escalas, as propriedades sensoriais são consideradas como fenômeno estático, ou seja, utiliza um único ponto de medida (DIJKSTERHUIS \& PIGGOTT, 2001). Porém, a percepção do aroma e do sabor é um fenômeno dinâmico, nesse sentido a técnica Tempo-intensidade é o melhor métodos para quantificar a resposta sensorial temporal (DIJKSTERHUIS, 1993). As mudanças dinâmicas na intensidade das percepções são mais evidentes em alimentos que sofrem transformações de fase na mucosa oral. Em geral, os alimentos requerem manipulação seqüencial com a língua e mastigação até ocorrer a deglutição (LEE III \& PANGBORN, 1986). Um mesmo composto pode ter distintos comportamentos temporais dependendo das características físico-químicas do alimento (MAILON \& EBERLER, 1997). Vários métodas tem sido aplicados para medir as mudanças naturais na percepção do sabor, porém a técnica mais completa e avançada é a análise Tempo-intensidade, a qual é capaz de avaliar a intensidade da percepção de qualquer gosto ou sabor no decorrer do tempo (CARDELlO, DA SILVA \& DAMÁSIO, 2001). Devido ao alto custo dos sistemas comerciais, CARDELLO et. al (2003) desenvolveram o sistema de coleta de dados Tempo-intensidade (SCDTI) que permitiu o desenvolvimento de várias pesquisas sensoriais usando esta metodologia no Brasil. No programa "Sistema de Coleta de Dados Tempo-Intensidade - SCDTI" para Windows, os provadores registram com auxílio do "mouse" a percepção de cada estímulo solicitado em função do tempo, para cada amostra. Os parâmetros das curvas T-I determinados pelo SCDTI são: tempo de intensidade máxima (TImax), intensidade máxima (Imax), tempo onde a intensidade máxima começa a declinar (Td), tempo de platô (Platô), área sob a curva (Área) e tempo total de duração do estímulo (Ttot) (CARDELlO, DA SILVA \& DAMÁSIO, 1999). A aplicação do método tempo-intensidade na pesquisa com edulcorantes auxiliou a investigação de modelos teóricos para a percepção de gostos avaliação, quantificação do gosto residual e caracterização dos edulcorantes (LARSON-POWERS \& PANGBORN, 1978; OVERBOSCH, 1986; CARDELLO, 1996; CARDELLO, SILVA \& DAMÁSIO, 1999). Há várias aplicações do método Tempo-intensidade além de edulcorantes, entre outros destacam-se: cerveja (amargor) e bebida de café (amargor), o estudo da textura em carnes, frutas (maciez e suculência), gomas, massas (coesividade) e molhos de salada (oleosidade), o estudo da adstringência e do amargor em bebidas como, por exemplo, o vinho e o estudo de aromas (géis saborizados) (JÚNIOR, TEIXEIRA \& AMBONI, 2007). O objetivo deste trabalho foi determinar o comportamento temporal de diferentes adoçantes em relação aos atributos gosto doce, gosto amargo e sabor de fruta (goiaba) em seis amostras de néctar de goiaba (Psidium Guajava L) adoçadas com 
sacarose e os edulcorantes: sucralose, ciclamato/sacarina 2:1, acessulfame-K, aspartame e estévia (Stévia rebaudiana Bertoni) .

\section{Material e Métodos}

\subsection{Material}

Foi utilizado base de néctar de goiaba (comercial), preparado conforme as instruções do fabricante. O néctar assim preparado, foi adoçado com cinco diferentes edulcorantes: aspartame puro (Nutrasweet); extrato de folhas de estévia (Steviafarma do Brasil); Mistura ciclamato / sacarina na

proporção de 2:1; sucralose (Splenda, Johnson), sacarose (Sigma Chemical Co.) e acessulfame-K (Slim diet).

\section{2 Preparo das amostras adoçadas com edulcorante}

Cada amostra foi adoçada, no momento das análises, com 9,6 \% de sacarose, concentração prédeterminada em teste de aceitação utilizando escala de ideal (BRITO, CÂMARA \& BOLINI 2007). Os edulcorantes foram adicionados em concentrações equivalentes a $10 \%$ de sacarose, determinadas pelo teste de estimação de magnitude. As concentrações de cada edulcorante foram as seguintes: 0,016\% de sucralose, $0,0360 \%$ da mistura ciclamato/sacarina $2: 1,0,0555 \%$ de acessulfame-K, $0,0555 \%$ de aspartame e $0,1 \%$ de estévia. As amostras foram preparadas com um dia de antecedência, mantidas a 6 $\pm 2^{\circ} \mathrm{C}$ e servidas (para avaliação sensorial) em temperatura de aproximadamente $10 \pm 2^{\circ} \mathrm{C}$. As avaliações sensoriais foram realizadas em cabines individuais e as amostras apresentadas em copos plásticos codificados com algarismos de três dígitos.

\subsection{Análise Sensorial}

Realizou-se a coleta de dados da análise tempo-intensidade pelo programa Sistema de Coleta de Dados Tempo-Intensidade (SCDTI), desenvolvido no Laboratório de Análise Sensorial da Faculdade de Engenharia de Alimentos da Universidade Estadual de Campinas (UNICAMP) (CARDELLO et al., 2003). Os estímulos doce, amargo e sabor de goiaba foram avaliados separadamente. 


\subsubsection{Seleção da Equipe de julgadores}

Efetuou-se a pré-seleção dos julgadores para a análise tempo-intensidade mediante testes triangulares de diferença, aplicados à análise seqüencial, e pela habilidade de trabalhar com o computador (MEILGAARD, CIVILLE \& CARR, 1999). Foi feito um intenso treinamento (4 a 6 sessões) com os provadores para familiarização com o programa e padronização do método. Na etapa de seleção, 17 julgadores pré-selecionados realizaram a análise tempo-intensidade com amostras de néctar de goiaba, adoçadas com os edulcorantes estudados a 10\% de equivalência de doçura em relação à sacarose. As amostras foram apresentadas de forma monádica com três repetições e avaliadas separadamente. A seleção dos julgadores foi realizada em função do poder de discriminação, repetibilidade e concordância com a equipe (DAMÁSIO \& COSTELL, 1991). Os julgadores que obtiveram valores de Famostra significativo $(p<0,30)$, Frepetição não-significativo $(p>0,05)$ e $F$ interação amostra $x$ julgador não-significativo $(p>0,05)$ em relação a cada parâmetro da curva foram selecionados. No total foram selecionados 15 julgadores que passaram por várias sessões de treinamento antes da realização dos testes finais.

\subsubsection{Programa Coleta de Dados - SCDTI}

Os dados tempo-intensidade foram coletados com programa desenvolvido no Laboratório de Análise Sensorial da Faculdade de Engenharia de Alimentos - UNICAMP, denominado "Sistema de Coleta de Dados Tempo-Intensidade - SCDTI" (CARDELLO et al.,2003). O SCDTI é um programa para coleta e manipulação de dados tempo-intensidade para computadores IBM-PC, ou compatíveis, que possui uma interface gráfica na forma de escala, na qual o usuário indica com auxílio do "mouse" a atual intensidade do estímulo a ser coletado e opera em ambiente "Windows". Possibilita a escolha do comprimento da escala a ser utilizada em cada teste, armazena a seqüência de dados lidos para uso e permite a manipulação dos dados armazenados. Os parâmetros das curvas T-I determinados foram: tempo de intensidade máxima (TImax), intensidade máxima (Imax), tempo onde a intensidade máxima começa a declinar (Td), tempo de platô (Platô), área sob a curva (Área) e tempo total de duração do estímulo (Ttot). O resultado é representado em forma de gráfico (Figura 1), que é sempre apresentado com o eixo horizontal representando os valores de tempo, com escala em segundos, e o eixo vertical representando os valores de intensidade. 


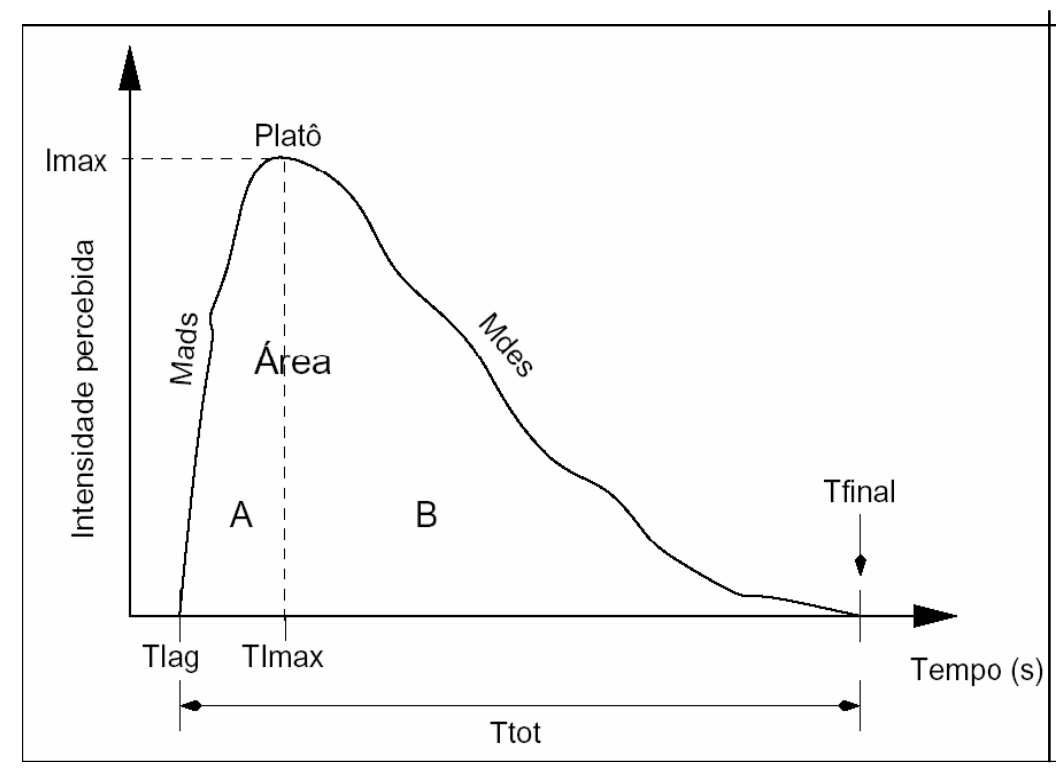

Figura 1 - Curva típica Tempo-Intensidade com alguns parâmetros representados. Valor de intensidade máxima (Imax), tempo para atingir a intensidade máxima (TImax), tempo total de duração do estímulo (Ttot), área total sob a curva (Área) tempo de duração da intensidade máxima (Platô), tempo antes do estímulo inicial (tempo de reação) (Tlag), tempo de leitura dos dados (Tfinal), taxa de crescimento (Mads), taxa de decrescimento (Mdes), área sob a curva antes da intensidade máxima (A), área sob a curva depois da intensidade máxima (B) (CLIFF \& HEYMANN, 1993; CARDELO, 1996).

\subsubsection{Avaliação sensorial das amostras}

$\mathrm{Na}$ análise tempo-intensidade as amostras foram apresentadas de forma monádica e balanceada, com três repetições, em copos plásticos de $50 \mathrm{~mL}$ codificados com algarismos de três dígitos. Padronizou-se a quantidade de amostra em $20 \mathrm{~mL}$ por teste, sendo os julgadores instruídos a colocar o volume total da amostra na boca e a seguir as instruções dadas pelo programa. Cada sessão foi iniciada pela escolha da opção mostrada na tela do computador, mediante pressão no "mouse". Ao primeiro aviso sonoro emitido, o julgador colocava toda a amostra na boca, ao segundo aviso o engolia e o terceiro aviso determinava o final do teste. A cada etapa o julgador indicava na escala, usando o "mouse", a intensidade do atributo sensorial avaliado. Os tempos de espera, permanência da amostra na boca e tempo total de duração dos testes foram padronizados e seguidos rigorosamente em todos os testes. O tempo de espera e permanência da amostra na boca foi de 10 segundos e o tempo total de duração dos testes variou em função da substância estudada, uma vez que a duração dos estímulos pode variar de acordo com a existência ou não de gosto residual. Padronizou-se o tempo de análise após a ingestão em 60 segundos para a doçura e amargor e 40 segundos para o sabor de goiaba. Utilizou-se escala estruturada de nove pontos no teste, cujo 0 corresponde a nenhum, 4,5 a moderado e 9,0 a forte. 
Após a coleta de dados em cada sessão de avaliação sensorial, o programa analisa e fornece os seguintes parâmetros: intensidade máxima registrada pelo julgador (Imax), tempo para atingir a intensidade máxima (Timax), tempo total de duração do estímulo (Ttot), área sob a curva tempointensidade (Área). Todos os provadores avaliaram todas as amostras

\subsubsection{Análise Estatística}

O delineamento experimental aplicado foi de blocos completos em relação aos provadores e blocos completos balanceados em relação às amostras, que foram apresentadas de forma monádica com três repetições.

Os dados coletados para os parâmetros citados foram submetidos à análise de variância univariada - ANOVA (fontes de variação: amostra e provadores) e interação para cada parâmetro. Aplicou-se o teste de Tukey para comparação das médias, sendo também efetuada a Análise de Componentes Principais. As análises estatísticas foram realizadas utilizando-se o programa Statistical Analysis System (SAS, 2001).

\section{Resultados e discussão}

\subsection{Análises Tempo Intensidade para o estímulo doce}

As curvas T-I para o estímulo doce está representada na Figura 2, os valores dos parâmetros encontram-se na Tabela 1. A partir dos parâmetros coletados para cada amostra e cada julgador foi realizada a análise de componentes principais (ACP), cujo resultado está apresentado na Figura 3. Verificou-se que 58,04\% da variação ocorrida entre as amostras foi explicada pelo primeiro eixo (Componente Principal 1), sendo que todos os parâmetros Imax, Ttot e Área contribuíram de forma praticamente equivalente para a variabilidade associada a esse eixo. $\mathrm{O}$ atributo Timax foi o que mais contribuiu para a variabilidade associada ao segundo eixo (Componente Principal 2). Os edulcorantes acessulfame-K e a mistura ciclamato/sacarina 2:1 evidenciaram de modo geral, os menores valores em todos os parâmetros das curvas tempo-intensidade. A sucralose caracterizou-se, principalmente, pelo parâmetro Timax. O aspartame caracterizou-se pelos parâmetros Imax e Área. A estévia apresentou

comportamento distinto das demais amostras, sendo caracterizada pelos atributos Ttot e Área. A proximidade das amostras com acessulfame-K, mistura ciclamato/sacarina 2:1 e sacarose indicam 
semelhanças desses edulcorantes no néctar de abacaxi para o estímulo doce. A estévia não apresenta proximidade com nenhuma amostra na figura bidimensional, o que sugere um estímulo doce peculiar.

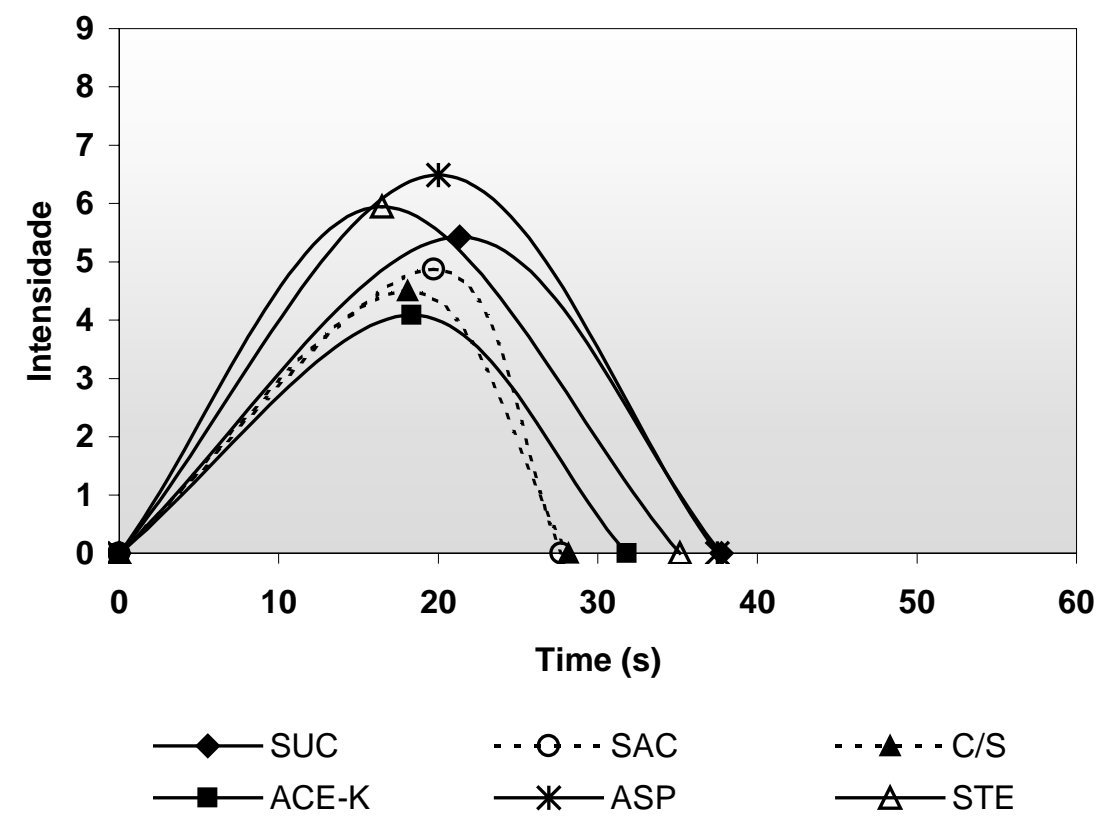

Figura 2. Curva Tempo Intensidade característica do gosto doce em néctar de goiaba (Psidium Guajava L.) adoçado com diferentes adoçantes

A análise de variância (ANOVA) evidenciou diferença significativa $(\mathrm{p} \leq 0,05)$ entre as amostras em relação a todos os parâmetros estudados. A Tabela 1 mostra a comparação das médias das amostras para cada um dos parâmetros, obtidas pelo teste de médias de Tukey $(p \leq 0,05)$. As características das curvas tempo-intensidade registradas para o estímulo doce são apresentadas, graficamente, na Figura 2. Para a construçãodas curvas foram utilizadas as médias dos parâmetros de cada amostra. O aspartame e a estévia apresentaram as maiores médias para o parâmetro Imax, sem revelar diferença significativa entre si $(\mathrm{p} \leq 0,05)$ O acessulfame-k, a mistura ciclamato/sacarina $2: 1$ e a sacarose (com médias intermediárias para Imax) não evidenciaram diferença significativa entre si, porém foram diferentes $(\mathrm{p} \leq 0,05)$ das demais amostras. $\mathrm{O}$ aspartame apresentou a maior média para o parâmetro intensidade máxima. Em relação ao Timax, a sucralose, aspartame e a sacarose apresentaram a maior média e a estévia a menor, diferindo significativamente das demais amostras. As amostras de acessulfame-k, a mistura ciclamato/sacarina $2: 1$ e a sacarose praticamente não diferiram significativamente entre si, apenas a sacarose possui Imax estatisticamente $(\mathrm{p} \leq 0,05))$ superior ao acessulfame-K . A curva do néctar de goiaba adoçado com aspartame evidenciou o maior tempo total de duração (Ttot), seguido 
pelas curvas da sucralose e estévia. Já a curva da amostra adoçada com sacarose e a mistura ciclamato/sacarina 2:1 apresentaram o menor tempo total de duração (Ttot).

Tabela 1. Média dos parâmetros das curvas Tempo Intensidade para o gosto doce em néctar de goiaba (Psidium Guajava L.) adoçado com diferentes adoçantes

\begin{tabular}{ccccc}
\hline Amostras & Timax (s) & Imax & Ttot (s) & Àrea \\
\hline SUC & $21,33^{\mathrm{a}}$ & $5,43^{\mathrm{b}}$ & $37,19^{\mathrm{a}}$ & $95,25^{\mathrm{b}}$ \\
SAC & $19,53^{\mathrm{ab}}$ & $4,87^{\mathrm{c}}$ & $27,71^{\mathrm{c}}$ & $73,84^{\mathrm{c}}$ \\
C/S & $18,09^{\mathrm{bc}}$ & $4,55^{\mathrm{cd}}$ & $29,53^{\mathrm{bc}}$ & $68,29^{\mathrm{c}}$ \\
ACE & $18,35^{\mathrm{bc}}$ & $4,20^{\mathrm{d}}$ & $31,82^{\mathrm{bc}}$ & $75,01^{\mathrm{c}}$ \\
ASP & $20,09^{\mathrm{ab}}$ & $6,39^{\mathrm{a}}$ & $37,56^{\mathrm{a}}$ & $122,74^{\mathrm{a}}$ \\
STE & $16,55^{\mathrm{c}}$ & $5,95^{\mathrm{a}}$ & $35,29^{\mathrm{a}}$ & $106,46^{\mathrm{b}}$ \\
\hline DMS & 2,1320 & 0,4792 & 3,1855 & 12,0370 \\
\hline
\end{tabular}

* valores da mesma coluna seguidos da mesma letra não apresentam diferença significativa $(\mathrm{p} \leq 0,05)$ de acordo com o teste de médias de Tukey DMS $=$ Diferença mínima significativa TImax $=$ Tempo para atingir a intensidade máxima. Imax $=$ Intensidade máxima do estímulo. Ttot $=$ Tempo total de duração do estímulo. Área $=$ área sob a curva

A estévia apresentou a menor média para Timax, diferindo significativamente $(\mathrm{p} \leq 0,05)$ das outras amostras, sugerindo uma maior rapidez para a percepção inicial do estímulo. Para a maioria dos parâmetros estudados em relação ao estímulo doce, a mistura ciclamato/sacarina 2:1 e o acessulfame-K foram os edulcorantes cujo perfil temporal mais se aproximou ao da sacarose em néctar de goiaba. A estévia apresentou o comportamento mais distinto em relação à sacarose e aos demais edulcorantes. A Figura 2 apresenta uma representação gráfica com todas as amostras e as respectivas variações de intensidade de percepção do estímulo ao longo do tempo que comprova esta semelhança. 


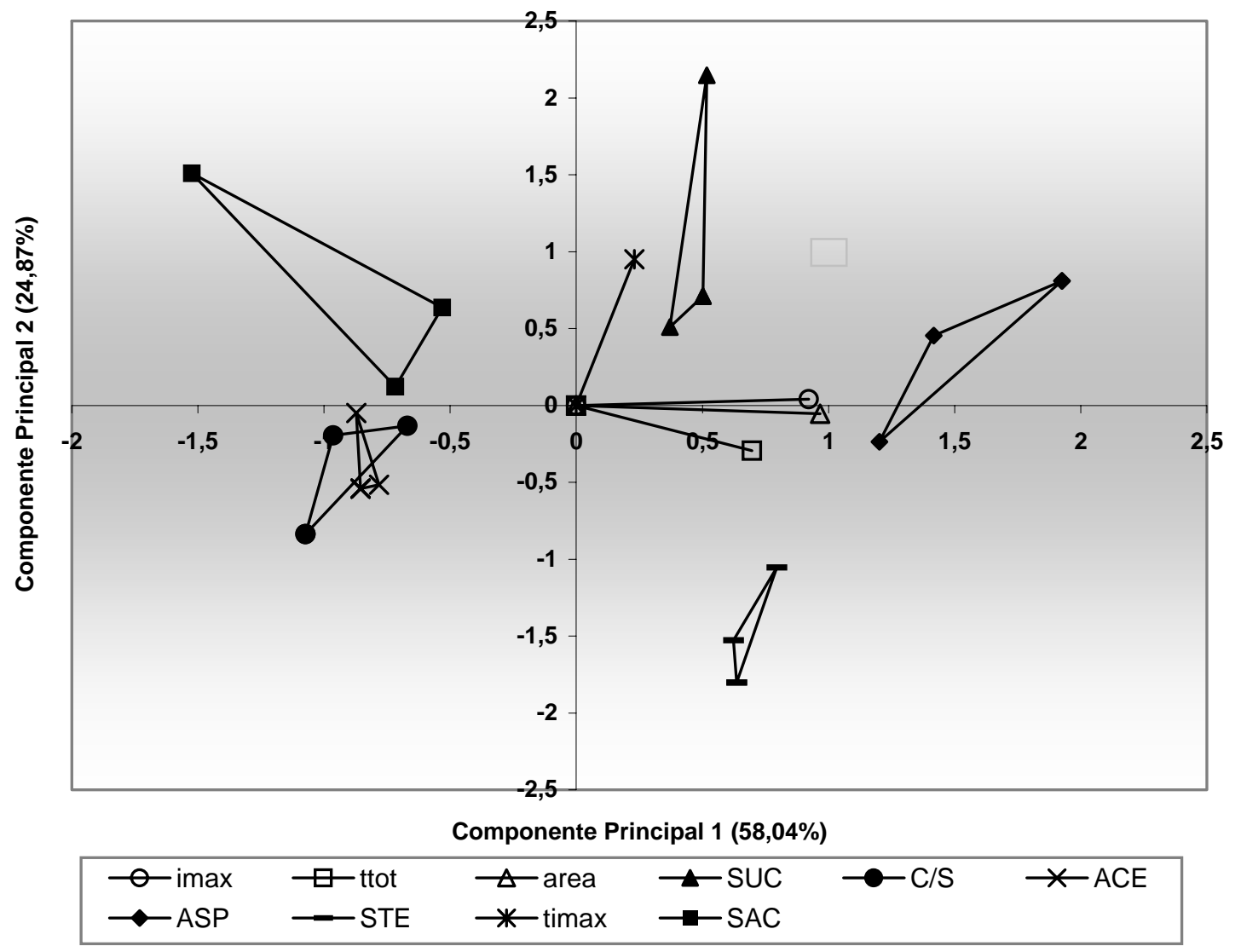

Figura 3 - Gráfico bidimensional da ACP dos parâmetros das curvas Tempo Intensidade para o estímulo doce em néctar de goiaba (Psidium Guajava L.) adoçado com diferentes adoçantes

Este resultado difere dos encontrados na literatura para outras amostras. Umbelino (2005) utlizando ciclamato/sacarina 2:1, aspartame, sacarose, sucralose e estévia na análise de suco e polpa de manga, verificou que o edulcorante com perfil sensorial temporal mais próximo ao da sacarose quanto ao estímulo doce foi o aspartame. O mesmo resultado foi encontrado por LARSON-POWERS \& PANGBORN (1978) que realizaram análise tempo intensidade de sacarose, aspartame, ciclamato e sacarina a $10 \%$ de equivalência de doçura em soluções aquosas e em bebidas sabor morango, laranja e limão e CARDELLO (1996) que avaliou o comportamento temporal para o estímulo doce de soluções aquosas de sacarose, aspartame, mistura ciclamato/sacarina $2: 1$ e estévia a $10 \%$ de equivalência de doçura. Marcellini (2005) na análise de suco de abacaxi encontrou semelhança significativa com a sacarose para os edulcorantes aspartame e sucralose para o estímulo doce, sugerindo a substituição da sacarose por estes edulcorantes sem comprometimento do sabor do suco de abacaxi. Ketelsen, Keay \& Wiet (1993) compararam através do método tempo-intensidade, amostras com diferentes edulcorantes e sacarose em meio tamponado e em soluções aquosas, concluíram que o meio influência a percepção temporal do estímulo doce. 


\subsection{Análises Tempo Intensidade para o estímulo amargo}

As curvas T-I para o estímulo amargo está representada na Figura 4, Os valores dos parâmetros encontram-se na Tabela 2. A partir dos parâmetros coletados para cada amostra e cada julgador foi realizada a análise de componentes principais (ACP), cujo resultado está apresentado na Figura 5. As amostras apresentaram boa repetibilidade como pode-se verificar pelas pequenas áreas dos triângulos formadas. Os componentes principais 1 e 2 explicaram juntos 99,35\% da variação ocorrida entre as amostras. Verificou-se que 93,11\% da variação foi explicada pelo primeiro eixo (CP1), sendo que os parâmetros Área, Ttot, Timax e Imax contribuíram de forma semelhante para a variabilidade associada a esse eixo. A sacarose, o aspartame e a sucralose ficaram em regiões próximas no gráfico bidimensional, não sendo caracterizados por nenhum parâmetro específico. As amostras com estévia, acessulfame-K e a mistura ciclamato/sacarina 2:1 estão mais próximas de todos os parâmetros da curva em relação às demais amostras, portanto são melhores caracterizadas por esses parâmetros. Estes edulcorantes não apresentam proximidades com nenhuma amostra na Figura bidimensionalm que sugere também um estímulo amargo peculiar das mesmas. A estévia apresentou comportamento distinto das demais amostras e caracterizou-se pelos parâmetros Área, Ttot, Imax e Timax, sugerindo a existência de gosto amargo residual.

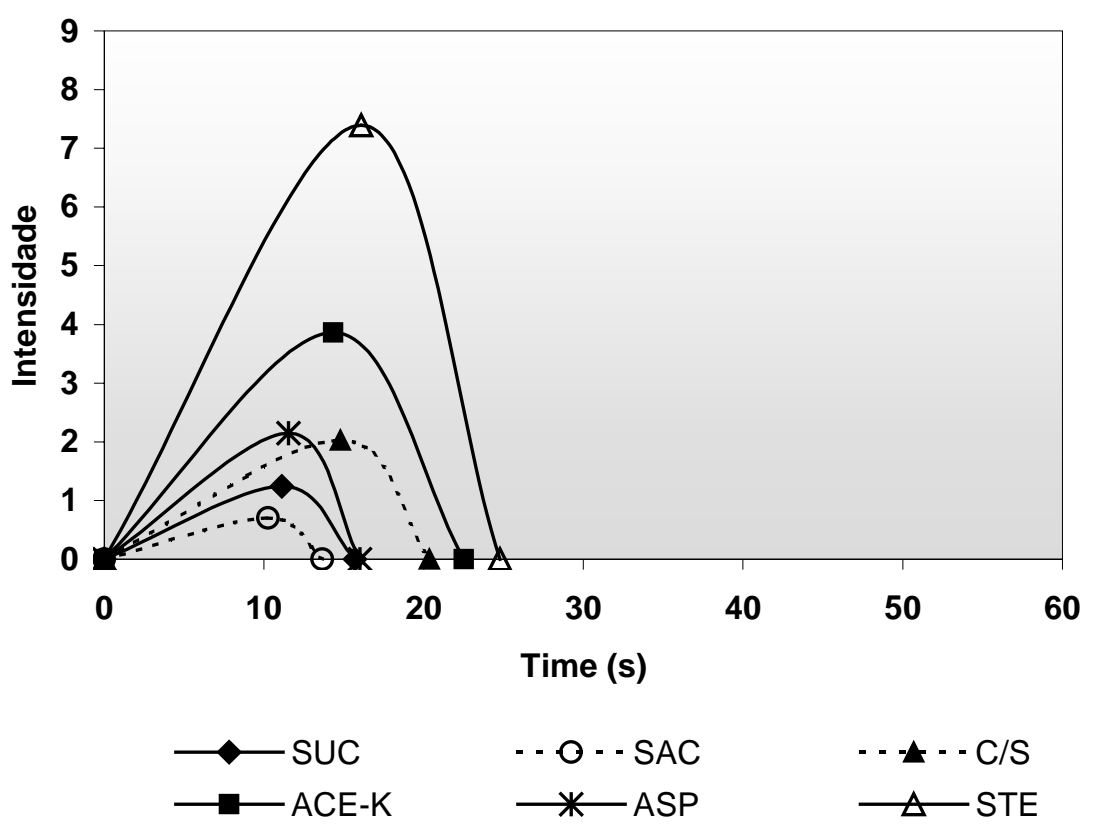

Figura 4. Curva Tempo Intensidade característica do gosto amargo em néctar de goiaba (Psidium Guajava L.) adoçado com diferentes adoçantes 
Tabela 2. Média dos parâmetros das curvas Tempo Intensidade para o gosto amargo em néctar de goiaba (Psidium Guajava L.) adoçado com diferentes adoçantes

\begin{tabular}{ccccc}
\hline Amostras & Timax (s) & Imax & Ttot (s) & Area \\
\hline SUC & $11,11^{\mathrm{cd}}$ & $1,24^{\mathrm{d}}$ & $15,70^{\mathrm{d}}$ & $15,21^{\mathrm{e}}$ \\
SAC & $10,26^{\mathrm{d}}$ & $0,70^{\mathrm{e}}$ & $13,65^{\mathrm{e}}$ & $6,77^{f}$ \\
C/S & $14,77^{\mathrm{b}}$ & $2,03^{\mathrm{c}}$ & $20,36^{\mathrm{c}}$ & $39,99^{\mathrm{c}}$ \\
ACE & $14,34^{\mathrm{b}}$ & $3,86^{\mathrm{b}}$ & $22,52^{\mathrm{b}}$ & $84,95^{\mathrm{b}}$ \\
ASP & $11,54^{\mathrm{c}}$ & $2,15^{\mathrm{c}}$ & $16,01^{\mathrm{d}}$ & $30,78^{\mathrm{d}}$ \\
STE & $16,08^{\mathrm{a}}$ & $7,39^{\mathrm{a}}$ & $24,77^{\mathrm{a}}$ & $140,50^{\mathrm{a}}$ \\
\hline DMS & 0,9356 & 0,1783 & 1,264 & 3,4269 \\
\hline
\end{tabular}

* valores da mesma coluna seguidos da mesma letra não apresentam diferença significativa $(\mathrm{p} \leq 0,05)$ de acordo com o teste de médias de Tukey DMS $=$ Diferença mínima significativa

TImax $=$ Tempo para atingir a intensidade máxima. Imax $=$ Intensidade máxima do estímulo. Ttot $=$ Tempo total de duração do estímulo.

Área $=$ área sob a curva

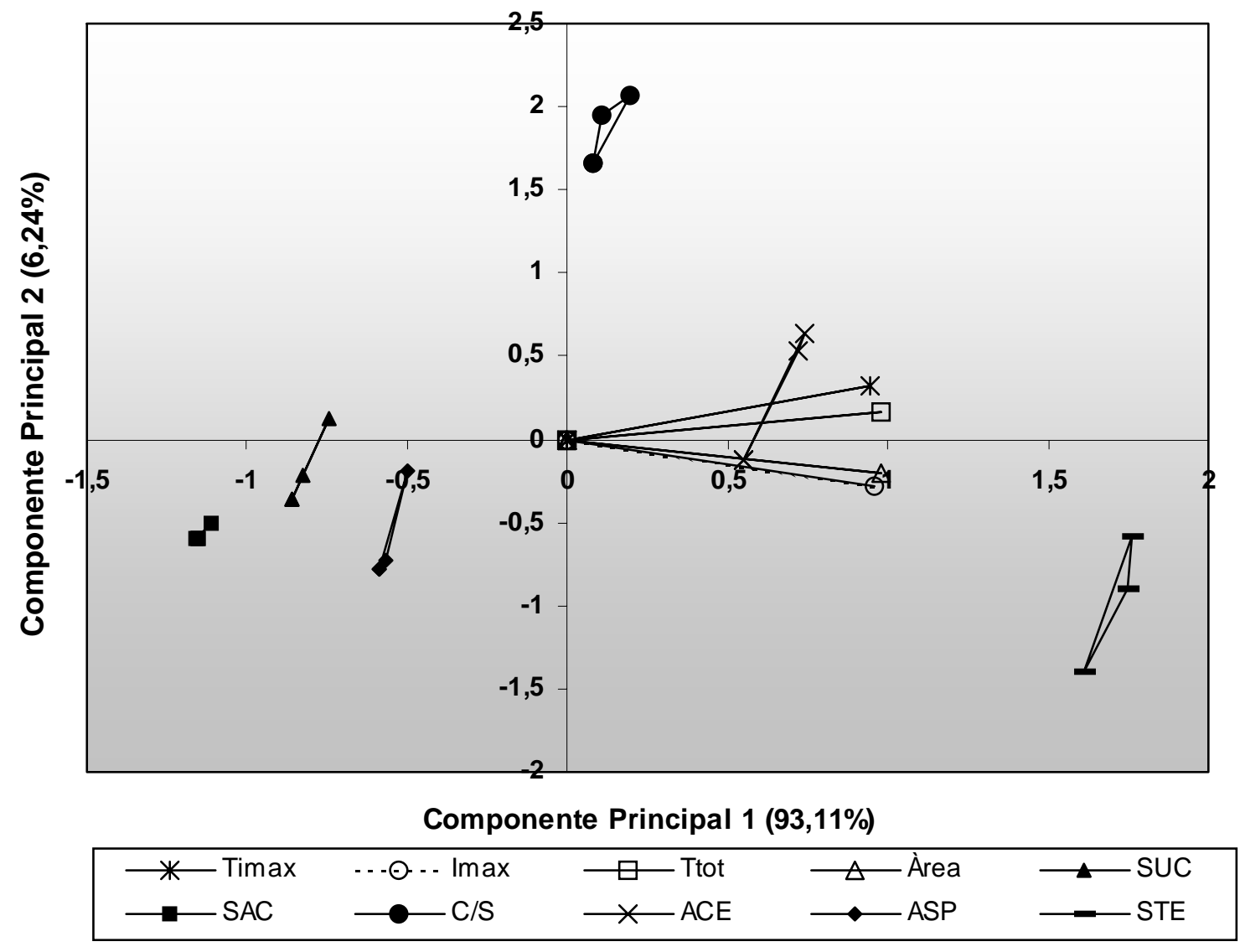

Figura 5 - Gráfico bidimensional da ACP dos parâmetros das curvas Tempo Intensidade para o estímulo amargo em néctar de goiaba (Psidium Guajava L.) adoçado com diferentes adoçantes 
A análise de variância revelou diferença significativa $(\mathrm{p} \leq 0,05)$ entre as amostras em relação a todos os parâmetros estudados. A Tabela 4 expressa os resultados do teste de médias de Tukey para o estímulo amargo em néctar de goiaba.

A estévia foi significativamente $(\mathrm{p} \leq 0,05)$ mais amarga do que as demais amostras (Tabela 2$)$, os valores absolutos para os parâmetros área e intensidade máxima foram praticamente duas vezes maiores que a segunda amostra mais amarga (acessulfame-k). O parâmetro Imax obedeceu a seguinte ordem crescente: sacarose, sucralose, mistura ciclamato/ sacarina 2:1, aspartame, acessulfame-K e estévia. Somente o aspartame e a mistura ciclamato/ sacarina 2:1 não diferiram $(\mathrm{p} \leq 0,05)$ entre si em relação a esse parâmetro. O maior tempo total de duração do estímulo (Ttot) foi observado para a estévia, seguida por acessulfame-k e a mistura ciclamato/sacarina 2:1 e o menor para a sacarose (todos com diferença significativa entre si). As amostras adoçadas com sacarose, sucralose e aspartame apresentaram as menores médias para o parâmetro Área, Ttot e Timax, indicando semelhanças temporais desses edulcorantes para o estímulo amargo em néctar de goiaba. A sacarose exibiu médias baixas para todos os parâmetros, não sendo possível representá-la graficamente. Em relação ao amargor, a sucralose e o aspartame foram os edulcorantes que mais se aproximaram da sacarose em néctar de goiaba.

Estes resultados corroboram com os obtidos por UMBELINO (2005) que encontrou um perfil sensorial similar da sacarose, sucralose e aspartame para o estímulo amargo em suco de manga e conclui que o aspartame foi o edulcorante que mais se aproximou da sacarose. Marcellini (2005) observou semelhanças temporais para os mesmos edulcorantes (sucralose e aspartame) com relação à sacarose no suco de abacaxi e a amostra com estévia foi a mais amarga, seguida pela amostra com a mistura ciclamato/sacarina 2:1. Larson-Powers \& Pangborn (1978) observaram que o perfil sensorial tempo-intensidade do aspartame para o estímulo amargo era indistinguìvel da sacarose e o ciclamato e a sacarina apresentararam amargor superior. Ott, Edwards e Palmer (1991) também verificaram a semelhança do aspartame em relação à sacarose para o estímulo amargo, em solução aquosa a 10\% de equivalência de doçura. Segundo CARDELLO (1996) e CARDELLO, SILVA e DAMÁSIO (1999) a estévia a 10\% de equivalência de doçura exibiu as maiores médias para os parâmetros Imax, Ttot, Área e Timax. Não houve diferença significativa $(p \leq 0,05)$ entre aspartame e a mistura ciclamato/sacarina 2:1 para o estímulo amargo, somente no parâmetro tempo de intensidade máxima. 


\subsection{Análises Tempo Intensidade para o estímulo sabor de fruta (sabor de goiaba)}

A análise de variância para o estímulo sabor de fruta em néctar de goiaba evidenciou diferença significativa $(\mathrm{p} \leq 0,05)$ entre as amostras em relação a todos os parâmetros. A Tabela 3 apresenta os resultados obtidos no teste de médias de Tukey. A Figura 7 mostra os resultados da análise de componentes principais (ACP) para o estímulo sabor de fruta em suco de goiaba. Os componentes principais 1 e 2 explicaram juntos 91,68\% da variação ocorrida entre as amostras. O primeiro eixo (CP1) explicou 69,92\% da variação e os parâmetros Ttot, Área e Imax constituíram os principais responsáveis por essa variação. O parâmetro Timax foi o que mais contribuíu para a variabilidade associada ao segundo eixo (CP2). O experimento apresentou ótima repetibilidade e as amostras distinguiram-se umas das outras. A sacarose caracterizou-se pelos parâmetros Área e Imax, a sucralose e aspartame por Timax. A estévia, acessulfame-K e a mistura ciclamato/sacarina 2;1 não se caracterizaram por nenhum parâmetro especificamente, estão distante de todas as amostras e dos parâmetros. A maior Imax foi observada para a sacarose, seguida pela sucralose e aspartame. A sacarose e o aspartame apresentaram as maiores médias para Área diferindo significativamente das outras amostras, mas não entre si. A estévia apresentou a menor média para todos os parâmetros, além de estar mais distante de todas as amostras e parâmetros na Figura bidimensional (Figura 7). As amostras com sacarose, sucralose e aspartame estão mais próximas de todos os parâmetros da curva em relação às outras amostras, portanto são melhores caracterizadas por esses parâmetros. A proximidade destas amostras sugere a semelhança dos mesmos para o estímulo sabor de goiaba. 

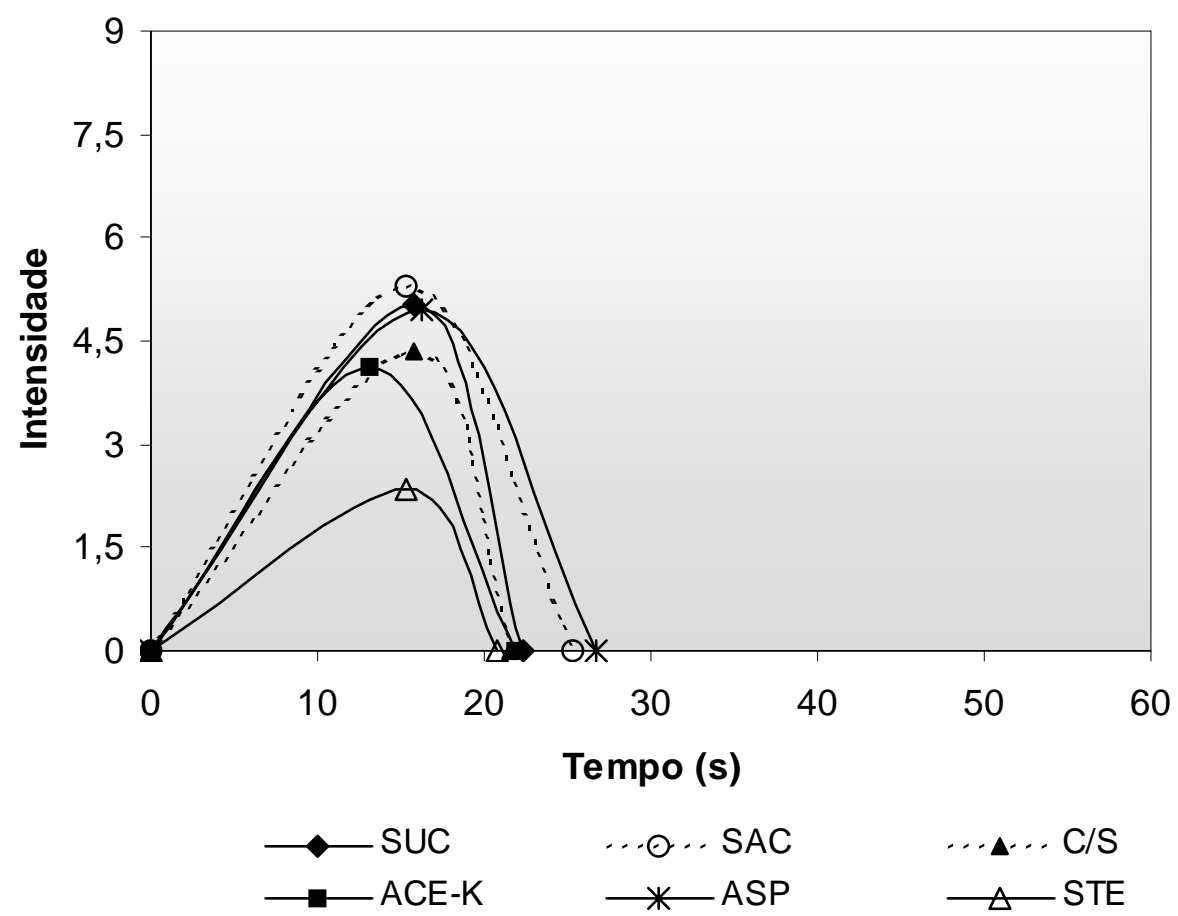

Figura 6. Curva Tempo Intensidade característica do sabor de fruta em néctar de goiaba (Psidium Guajava L.) adoçado com diferentes adoçantes

Tabela 3. Média dos parâmetros das curvas Tempo Intensidade para o sabor de fruta em néctar de goiaba (Psidium Guajava L.) adoçado com diferentes adoçantes

\begin{tabular}{ccccc}
\hline Amostra & Timax (s) & Imax & Ttot (s) & Area \\
\hline SUC & $15,84^{\mathrm{ab}}$ & $5,02^{\mathrm{ab}}$ & $22,29^{\mathrm{c}}$ & $71,22^{\mathrm{b}}$ \\
SAC & $15,25^{\mathrm{b}}$ & $5,29^{\mathrm{a}}$ & $25,25^{\mathrm{b}}$ & $78,19^{\mathrm{a}}$ \\
C/S & $15,84^{\mathrm{ab}}$ & $4,33^{\mathrm{c}}$ & $21,57^{\mathrm{cd}}$ & $52,49^{\mathrm{d}}$ \\
ACE & $13,14^{\mathrm{c}}$ & $4,14^{\mathrm{c}}$ & $21,88^{\mathrm{c}}$ & $58,35^{\mathrm{c}}$ \\
ASP & $16,31^{\mathrm{a}}$ & $4,96^{\mathrm{b}}$ & $26,69^{\mathrm{a}}$ & $81,54^{\mathrm{a}}$ \\
STE & $15,24^{\mathrm{b}}$ & $2,35^{\mathrm{d}}$ & $20,77^{\mathrm{d}}$ & $33,92^{\mathrm{e}}$ \\
\hline DMS & 0,8094 & 0,3225 & 0,9850 & 3,6564 \\
\hline
\end{tabular}

* valores da mesma coluna seguidos da mesma letra não apresentam diferença significativa $(\mathrm{p} \leq 0,05)$ de acordo com o teste de médias de Tukey DMS $=$ Diferença mínima significativa TImax $=$ Tempo para atingir a intensidade máxima. Imax $=$ Intensidade máxima do estímulo. Ttot $=$ Tempo total de duração do estímulo. Área $=$ área sob a curva 


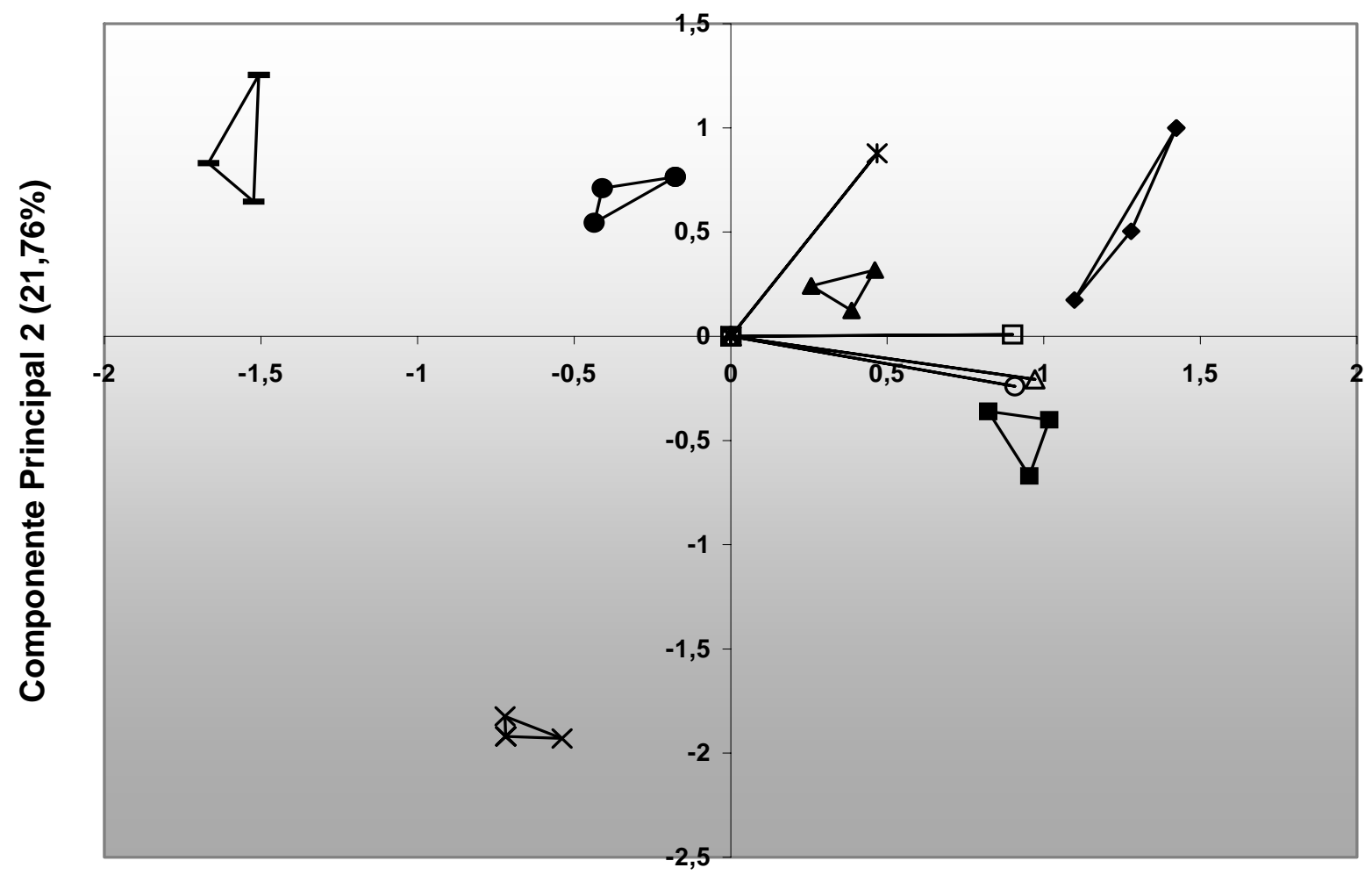

Componente Principal 1 (69,92\%)

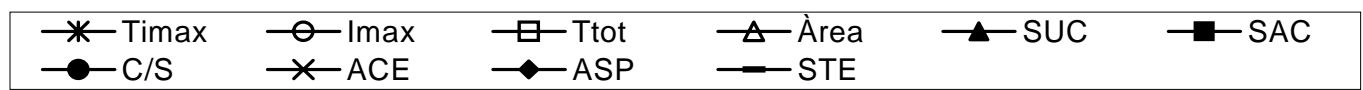

Figura 7 - Gráfico bidimensional da ACP dos parâmetros das curvas Tempo Intensidade para o estímulo sabor de fruta em néctar de goiaba (Psidium Guajava L.) adoçado com diferentes adoçantes

As amostras adoçadas com sacarose apresentaram a maior intensidade máxima (Imax) para o estímulo sabor de fruta, enquanto o aspartame exibiu o maior tempo total de duração do estímulo (Ttot) em néctar de goiaba. Apesar da diferença de magnitude na percepção do estímulo, essa característica pode ser interpretada como intensificação do sabor de goiaba pelo aspartame em relação à sacarose. Estudos evidenciaram a capacidade do aspartame para intensificar o sabor de fruta em bebidas, Baldwin \& Korschgen (1979) observaram intensificação do sabor de fruta nas bebidas sabor cereja e laranja adoçadas com aspartame em comparação com as adoçadas com sacarose. Matysiak \& Noble (1991) verificaram que o aspartame exibiu tempo total de duração do estímulo (Ttot) superior em relação aos estímulos doçura e sabor de fruta e a estévia apresentou os menores valores de Imax e Ttot. 
Pela análise de variância verifica-se que a amostra com estévia obteve menores valores significativamente $(\mathrm{p} \leq 0,05)$ para os parâmetros intensidade máxima, área e tempo total de duração do estímulo, sugerindo a possibilidade de mascaramento do estímulo sabor de goiaba. Este fato se deve provavelmente à presença de amargor ou amargor residual deste edulcorante, o que possibilita uma descaracterização do néctar de goiaba por este edulcorante. Resultado este confirmado por MARCELLINI (2005) em suco de abacaxi e em suco de manga por UMBELINO (2005).

\title{
5. Conclusão
}

Nas curvas tempo-intensidade para o estímulo doce em néctar de goiaba, a estévia apresentou a menor média para Timax, diferindo significativamente $(\mathrm{p} \leq 0,05)$ das outras amostras, sugerindo uma maior rapidez para a percepção inicial do estímulo. Para a maioria dos parâmetros estudados em relação ao estímulo doce, a mistura ciclamato/sacarina 2:1 e o acessulfame-K foram os edulcorantes cujo perfil temporal mais se aproximou ao da sacarose quanto ao estímulo doce. Em relação ao estímulo amargo, A estévia foi significativamente $(\mathrm{p} \leq 0,05)$ mais amarga do que as demais amostras, e apresentou maior tempo total de duração do estímulo (Ttot), confirmando a presença de amargor residual intenso na amostra. de de Com relação à este estímulo, a sucralose e o aspartame foram os edulcorantes que mais se aproximaram da sacarose em néctar de goiaba. Para o estímulo sabor de fruta, a sacarose exibiu a maior intensidade máxima e o aspartame o maior tempo total de duração do estímulo, indicando potencialização do sabor de fruta na amostra adoçada com esse edulcorante. A sucralose também apresentou perfil sensorial semelhante à sacarose quanto ao estímulo sabor de goiaba, sendo desaconselhável o uso de estévia, que devido ao seu amargor, descaracteriza o néctar de goiaba.

\begin{abstract}
The objective of this study, was determine the temporal characteristics of sweetness, bitterness and fruitiness on guava nectar (Psidium Guajava L.) sweetned with sucrose and five edulcorants (sucralose, ciclamato/sacarina 2:1, acessulfame-k, aspartame e estévia (Stévia rebaudiana Bertoni) in equi-sweet of sucrose at 10\%. Time-intensity (T-I) method was used and the time-intensity curves for each substance were made with the software "Sistema de Coleta de Dados Tempo-Intensidade SCDTI" for Windows. The judges registered the perception of each stimuli as function of time for each sample using the mouse. The sample sweetened with stevioside showed maximal intensity for sweet and bitter tastes and these characteristic persisted for a long period, indicating intense sweet and bitter aftertaste. The sample with sucrose displayed a clean sweet taste with no unpleasant aftertaste. Sucrose had a higher intensity of fruit flavour. Although aspartame elicited significantly longer persistence of fruitiness, suggesting an intensification effect on fruitiness of guava juice. The sensorial profiles of aspartame were similar to that of sucrose in the time intensity analysis.
\end{abstract}

Key-words: guava, nectar, time intensity, edulcorant. 


\section{Referências bibliográficas}

BRITO, C.A.K. ; CÂMARA, V.H.A.; H.M.A. BOLINI Equivalência de dulçor e poder edulcorante de néctares de goiaba adoçados com diferentes edulcorantes. Revista Brasileira de Tecnologia Agroindustrial, v.01, n.02, p.26-36, 2007.

CARDELLO, H.M.A.B., DA SILVA, M.A.A.P., DAMÁSIO, M.H.; LOBÃO, F. Programa Sistema de Coleta de Dados Tempo-Intensidade. Boletim da Sociedade Brasileira de Ciência e Tecnologia de Alimentos, v.37, supl., p.54-60, 2003.

CARDELLO, H.M.A.B., DA SILVA, M.A.A.P., DAMÁSIO, M.H.; Parâmetros de curvas tempo-intensidade dos estímulos doce e amargo de edulcorantes: seleção por análise discriminante por passos e análise de correlação. Alimentos e Nutrição, v.12, p.11-26, 2001.

CARDELLO, H.M.A.B.; SILVA, M. A.; DAMÁSIO, M.H. Análise tempointensidade dos estímulos doce e amargo de extrato de folhas de estévia (Stévia rebaudiana Bertoni) em doçura equivalente à sacarose em solução a $10 \%$. In: ALMEIDA, T.C.A.; HOUGH, G.; DAMÁSIO, M.H.; DA SILVA, M.A.A.P. Avanços em análise sensorial. São Paulo: Varella, 1999. p. 199-212.

CARDELLO, H.M.A.B. Caracterização sensorial de aspartame, ciclamato/sacarina 2:1 e extrato de folhas de estévia (Stévia rebaudiana Bertoni): equivalências em doçura, análise descritiva quantitativa e análise tempo-intensidade. Tese de Doutorado. Universidade Estadual de Campinas. Faculdade de Engenharia de Alimentos. Campinas, SP, 1996, $237 \mathrm{p}$.

CLIFF, M.; HEYMANN, H. Development and use of time-intensity methodology for sensory evaluation: a review.

Food Research International, v. 26, p. 375-385, 1993.

DAMÁSIO, M. H.; COSTELL, E. Análisis sensorial descriptivo: generación de descriptores y selección de catadores. Revista Agroquímica e Tecnologia de Alimentos., v.31,n.2, p.165-178, 1991.

DIJKSTERHUIS, G. B.; PIGGOTT, J. R. Dynamic methods of sensory analysis. Trends in Food Science \& Technology, v. 11, p. 284-290, 2001.

DIJKSTERHUIS, G. B. Principal component analysis of time-intensity bitterness curves. Journal of Sensory Studies, v.8, n. 4, p. 317-328, 1993.

FORDE, C. G. DELAHUNTY, C.M. Understanding the role cross-modal sensory interactions play in food acceptability in younger and older consumers. Food Quality and Preference, v. 15, p. 715-727, 2004.

JÚNIOR, N.G.C.; TEIXEIRA, E.; AMBONI, R. D.M.C. Método Tempo-intensidade: Revisão. Boletim Ceppa, v. 25, n. 1 , p. $1-14,2007$

KETELSEN, S.M.; KEAY, C.L.; WIET, S.G. Time-intensity parameters of selected carbohydrate and high potency sweetners. Journal of Food Science, v. 58, p. 1418-1421, 1993.

LARSON-POWERS, M.; PANGBORN, R. M. Pairs comparison and time-intensity measurements of sensory properties of beverages and gelatins containing sucrose or synthetic sweeteners. Journal of Food Science, v. 43, p. 4146, 1978.

LEE III, W. E.; PANGBORN, R. M. Time-intensity: the temporal aspects of sensory perception. Food Technology, v. 40, p. 71-82, 1986.

MAILON, V.S.; EBERLER, S.E. Time-intensity measurement of matrix effects on retronasal aroma perception. Journal of Sensory Studies, v.12, p. 303-316, 1997.

MARCELLINI, P.S. Caracterização sensorial por perfil livre e análise tempo-intensidade de suco de abacaxi (Ananas comosus L. Merril) reconstituído e adoçado com diferentes edulcorantes. Tese de Doutorado. Universidade Estadual de Campinas. Faculdade de Engenharia de Alimentos. Campinas, SP. 2005. 85p. 
MEILGAARD, M.; CIVILLE, G.V.; CARR, B.T. Sensory Evaluation Techniques. New York: Boca Raton, 3 ed. 1999. 387p.

MUÑOZ, A.M.; CIVILLE, G.V.; CARR, B.T. Sensory evaluation in quality control. New York: Van Nostrand Reinhold, 1992, 240 p.

OTT, D. B.; EDWARDS, C. L.; PALMER, S. J. Perceived taste intensity and duration of nutritive and non-nutritive sweeteners in water using time-intensity (T-I) evaluations. Journal of Sensory Studies, v. 56, p. 535-542,1991.

OVERBOSCH, P.; ENDEN, J. van den; KEUR, B. An improved method for measuring perceived intensity/time relationships in human taste and smell. Chemical Senses, v. 11, n. 3, p. 331-338, 1986.

SAS Institute. SAS User's Guide: statistics. Cary, USA: SAS Inst., 2001.

UMBELINO, D.C. Caracterização sensorial por análise descritiva quantitativa e análise tempo-intensidade de suco e polpa de manga (Magnífera indica L.) adoçados com diferentes edulcorantes. Tese de Doutorado. Universidade Estadual de Campinas. Faculdade de Engenharia de Alimentos. Campinas, SP. 2005. 190p.

\section{Agradecimentos}

financeiro.

À CNPq- Conselho Nacional de Desenvolvimento Científico e Tecnológico pelo apoio

Nome Completo: *Carlos Alexandre Koguishi de Brito Helena Maria André Bolini

Filiação Institucional: Faculdade de Engenharia de Alimentos - Universidade Estadual de Campinas UNICAMP

Departamento: Alimentos e Nutrição (Laboratório de Análise Sensorial)

Função ou cargo ocupado: Aluno de pós graduação e *docente (orientadora)

Endereço para correspondência: Rua Monteiro Lobato, 80 Cidade Universtiária "Zeferino Vaz Dias" Barão Geraldo Campinas -SP C.P. 6121 CEP: 13083-862.

Telefone para contato: *(19) 3521-4059

e-mail: brito@fea.unicamp.br 